\title{
Efecto Spillover en los cuidadores de infantes de 1.5 a 5 años durante la pandemia del COVID-19
}

\author{
Spillover effect in caregivers of 1.5 to 5 year old infants during \\ the COVID-19 pandemic
}

\author{
Demian A. Herrera Morban', Jorge Massiel Méndez', Carlos Sosa'
}

\section{RESUMEN}

Introducción: El infante percibe y reproduce las emociones de los padres, este efecto es conocido como la teoría de Spillover. El objetivo del presente estudio fue correlacionar el grado de ansiedad de los padres con el grado de ansiedad/depresión en los hijos, de 1,5 a 5 años de edad, durante la pandemia del SARS-COV2. Materiales y Métodos: Se realizó un estudio descriptivo de corte transversal a través de un formulario en línea con un muestreo en bola de nieve Para medir el nivel de ansiedad de los cuidadores/padres/madres se utilizó la escala de ansiedad de BECK y para medir el nivel de ansiedad de los infantes los acápites de ansiedad del Cuestionario de Comportamiento Infantil de 1.5 a 5 años. Los datos fueron analizados en SPS.S La correlación entre ansiedad de los padres y de los hijos se realizó por la Correlación de Spearman. Se solicitó el consentimiento informado de los participantes. Resultados: Fueron incluidos 29 padres-cuidadores, quienes devolvieron el cuestionario con las respuestas. El 72,4\% de los padres cuidadores presentaron ansiedad moderada a severa. La correlación entre ansiedad de los padres y la de los hijos fue de 0,435 $p=0,018$. La relación entre el nivel de ansiedad/depresión de los niños con el apego ansioso del infante fue de $0.653 \mathrm{p}=0,0001$. Conclusiones: Nuestros hallazgos sugieren un alto nivel de ansiedad en los cuidadores. La correlación con la ansiedad en los niños fue moderada. La ansiedad depresión en los niños se relacionó con el apego ansioso.

Palabras clave: Ansiedad, cuidadores, conducta, temperamento, niños.

\begin{abstract}
Introduction: Infants perceive and reproduce the emotions of their parents, this effect is known as the Spillover theory. The objective of the present study was to correlate the degree of anxiety in parents with the degree of anxiety / depression in children aged 1.5 to 5 years old, during the SARS-COV2 pandemic. Materials and Methods: This was a descriptive, cross-sectional study carried out through an online form with a snowball sampling. To measure the level of anxiety in caregivers / parents, the BECK anxiety scale was used and to measure the level of anxiety in infants we used the anxiety sections of the Child Behavior Questionnaire from 1.5 to 5 years. Data were analyzed in SPS.S The correlation between parental and child anxiety was made by the Spearman Correlation. Informed consent of the participants was requested. Results: 29 parents-caregivers who answered the questionnaire were included. $72.4 \%$ of the caregiver / parents presented moderate to severe anxiety. The correlation between parental and children's anxiety was $0.435 \mathrm{p}=0.018$. The relationship between the children's anxiety / depression level with the infant's anxious attachment was $0.653 \mathrm{p}=0.0001$. Conclusions: Our findings suggest a high level of anxiety in caregivers. The correlation with anxiety in children was moderate. Anxiety and depression in children were related to anxious attachment.
\end{abstract}

Key words: Anxiety, caregivers, behavior, temperament, children.

\footnotetext{
${ }^{1}$ Hospital Pediátrico Dr. Hugo Mendoza. Centro de Investigación. Santo Domingo, República Dominicana.

Correspondencia: Demian A. Herrera Morban Correo: herreramorbanmd@gmail.com

Conflicto de Interés: Los autores declaran no poseer conflicto de interés

Recibido: 01/06/2020 Aceptado:29/06/2020

Doi: https://doi.org/10.31698/ped.47022020005
}

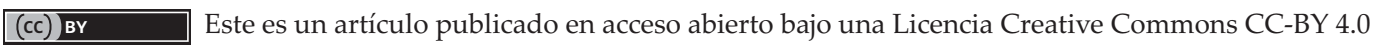




\section{INTRODUCCIÓN}

La pandemia secundaria al novel coronavirus (SARS-COV2) ha afectado 213 países generando 1.9 millones de infectados. Con el objetivo de controlar el impacto de la pandemia se han implementado medidas sociales desde el distanciamiento social como lo más leve hasta la cuarentena ${ }^{(1)}$, esto ha causado un aumento en la aparición, agravamiento de enfermedades mentales secundarios al estrés generado por la situación, causando hasta violencia doméstica $^{(2)}$. La salud mental de los cuidadores influye en el desarrollo infantil, en la respuestas de los cuidadores a los infantes y en el apego de los infantes a los cuidadores ${ }^{(3)}$, ésta a su vez guarda relación entre la interacción entre ambos cuidadores y entre los cuidadores con el medio ambiente ${ }^{(4)}$.

El estatus socio-económico bajo, incrementa el riesgo a exponerse a eventos/experiencias negativas relacionadas con el entorno donde viven, como pueden ser el desorden o inestabilidad familiar; estos eventos negativos en la vida generan un disregulación biológica similar a un estado inmune inflamatoria que conlleva a cambios epigenéticos en el neurodesarrollo de manera negativa, también crea el efecto de apagado, que disminuye la percepción de estímulos nocivos del entorno e incluye los estímulos adecuados, afectando los eje social y del lenguaje $\mathrm{e}^{(5-11)}$, estas señales de retroalimentación intervienen con la maduración y polarización neuronal adecuada para el neurodesarrollo ${ }^{(12)}$; por lo cual están relacionadas con el desarrollo de trastornos mentales en el adulto ${ }^{(13)}$.

El conflicto y estrés entre los cuidadores modifica las prácticas de respuesta y cuidado a los infantes ${ }^{(14)}$, afectando el apego posteriormente ${ }^{(3)}$; estos agravantes son incrementados durante la cuarentena secundaria a la pandemia, aumentando la exposición al infante de eventos los cuales son más propensos a copiar y adoptar ${ }^{(15)}$. El infante percibe y reproduce las emociones de los padres, respondiendo de manera negativa empeorando la situación ${ }^{(16,17)}$, este efecto es conocido como la teoría de Spillover. El objetivo del estudio fue correlacionar el nivel de ansiedad de los padres y ansiedad de los hijos durante la cuarentena impuesta por la pandemia delSARS-COV2.

\section{MATERIALES Y MÉTODOS}

Se realizó un estudio descriptivo de corte transversal con componente analítico. La hipótesis alterna evaluada fue que los niveles de ansiedad de los padres pueden ser transmitidos a los hijos. Para medir el nivel de ansiedad de los cuidadores/padres/madres se utilizó la escala de ansiedad de BECK y para medir el nivel de ansiedad de los infantes se utilizó las 14 preguntas del Cuestionario de Comportamiento Infantil de 1.5 a 5 años (CBCL 1.5-5) que evalúan ansiedad/depresión, y para el apego ansioso se evaluaron 8 preguntas de los 14 ítem sobre ansiedad/depresión que evalúan el apego del infante con su padre. El muestreo fue en bola de nieve. El cuestionario fue suministrado a través de un enlace de la plataforma de Google Forms. Los participantes completaron el cuestionario entre el 27 de abril y el 11 de mayo del 2020. Los investigadores y las partes interesadas promovieron la encuesta actuando como guardianes en las redes sociales y apegándose al Checklist for Reporting Results of Internet ESurveys ${ }^{(16)}$.

Los criterios de inclusión requerían que los participantes fueran binomio de cuidadores independiente del género de infantes de 1,5 a 5 años sanos. Los criterios de exclusión fueron antecedentes de parálisis cerebral infantil, trastorno del espectro autista, trastorno de déficit de atención e hiperactividad, epilepsia o convulsiones, trastorno del habla o lenguaje, antecedentes de enfermedad mental.

Las variables estudiadas relacionadas al niño/a fueron grupo etario, genero, orden de nacimiento, $y$ el nivel de ansiedad / depresión de acuerdo al CBCC 1,5 -5. (Child Behavior check list) Los datos de los padres: se consignó según quien suministro, grupo etario, escolaridad, estado marital, número de hijos y los niveles de ansiedad de acuerdo al cuestionario BECK y el nivel socioeconómico este último atendiendo al ingreso per cápita diario y el ajustados por paridad de poder adquisitivo en dólares americanos, de manera que: Pobres: $<\mathrm{USD} \$ 4$, Vulnerables: USD\$4-10, Clase media: USD10-50 y Residual:>USD\$50).

El análisis de los datos se realizó con el programa estadístico SPSS V 23. Las variables cualitativas se 
expresan en porcentajes. Para determinar la relación entre el nivel de ansiedad de los padres-cuidadores, se utilizó la correlación de Spearman.

El estudio se sometió a revisión del Comité de Ética del Hospital Pediátrico Dr. Hugo Mendoza quien posterior a su revisión otorgo la aprobación de ética. Como primer acápite del cuestionario se otorgó el consentimiento informado, en caso de negarse el cuestionario no permitía el llenado.

\section{Definición de los instrumentos}

Cuestionario de BECK El Inventario de ansiedad de Beck es un cuestionario de autoinforme creado por el psicólogo Aaron T. Beck que consiste en 21 preguntas con modalidad de selección múltiple. Las preguntas utilizadas remiten hacia los síntomas más comunes de ansiedad que el sujeto haya podido experimentar durante la última semana y enfatiza en síntomas tales como temblores, sudoración, escenas temidas, entre otros.

Cuestionario $C B C L$ ( 1,5 a 5$)$ : consiste en un formulario de informe médico usado ampliamente la identificación de los problemas de comportamiento en los niños. En esa versión los padres u otras personas que interactúan con el niño califican su conducta en una escala de 3 puntos (no es cierto, un poco o a veces cierto y muy cierto o, a menudo cierto). Para el apego ansioso se evaluaron 8 preguntas de los 14 ítem sobre ansiedad/depresión que evalúan el apego del infante con su padre.

\section{RESULTADOS}

29 cuidadores llenaron el cuestionario durante el periodo. Los datos fueron suministrados por la cuidadora-madre en el $89,7 \%(26 / 29)$ y por el cuidador padre en el 10,3\% (3/29). En relación al número de hijos 14/29 tenían un solo hijo, 9/29, 2 y $6 / 29$, 3. Las características demográficas de los padres/madres cuidadores se detallan en la Tabla 1.

Los resultados de la situación socioeconómica, así con el nivel de ansiedad de los padres cuidadores se detallan en la Tabla 2.
Tabla 1. Características demográficas de los padres /madres cuidadores $\mathrm{N}=29$

\begin{tabular}{lll}
\hline Datos de la cuidadora -madres & N & $\%$ \\
\hline Grupo etario & & \\
$25-35$ & 10 & $(34,5)$ \\
$36-40$ & 12 & $(41,4)$ \\
$51-60$ & 7 & $(24,1)$ \\
Escolaridad & & \\
$\quad$ Secundaria & 2 & $(6,9)$ \\
Universitaria & 27 & $(93,1)$ \\
Datos del cuidador -padre & & \\
Grupo etario & & \\
$25-35$ & 9 & $(31)$ \\
$36-45$ & 20 & $(69)$ \\
Escolaridad & & \\
Primaria & 1 & $(3)$ \\
Secundaria & 4 & $(14)$ \\
Universitaria & 24 & $(83)$ \\
Situación marital de los cuidadores/as & & \\
$\quad$ Casados/ unión libre & 27 & $(93)$ \\
Divorciados/separados & 2 & $(7)$ \\
\hline
\end{tabular}

Tabla 2. Estado socioeconómico de los cuidadores y nivel de ansiedad de acuerdo al puntaje del cuestionario BECK. $\mathrm{N}=29$

\begin{tabular}{lll}
\hline ¿Cuál es el rango de ingresos que percibe tu hogar? & $\mathbf{N}$ & $\mathbf{\%}$ \\
\hline Pobres & 5 & 17.2 \\
Vulnerables & 3 & 10.3 \\
Media & 17 & 58.6 \\
Residual & 4 & 13.8 \\
Ansiedad de padres & & \\
Ansiedad muy baja (0-21) & 8 & 27.6 \\
Ansiedad moderada (22-35) & 13 & 44.8 \\
Ansiedad severa (36 o más) & 8 & 27.6 \\
\hline
\end{tabular}

Se analizó la correlación entre el nivel de depresión/ansiedad de los padres y las del niño por medio de la correlación Spearman cuyo resultado fue Rhof $=0,435 p=0,018$. El nivel de ansiedad y depresión en los niños correlacionó con apego ansioso Rho $=0,648 \mathrm{p}=0,0001$.

\section{DISCUSIÓN}

Los infantes copian y reproducen las emociones que presentan sus cuidadores, siendo las de carácter negativo las replicadas frecuentemente $e^{(14,15,17,18)}$. Un porcentaje mayoritario de los padres presentaron ansiedad depresión entre moderada a severa. Esta situación podría estar relacionado con la cuarentena 
por la pandemia, y sus efectos sobre la economía y la salud mental ${ }^{(2)}$. Se encontró una correlación positiva moderada entre la ansiedad de los padres y la de los hijos.

Las características sociodemográficas de los cuidadores pueden influir en la respuesta al estrés por la cuarentena ${ }^{(2)}$, a su vez modificar la interacción entre los cuidadores y los infantes, lo que afecta el apego en vez de generar ansiedad en los infantes ${ }^{(3-11)}$.

La correlación entre el nivel de ansiedad de los niños y el apego ansioso (niños preocupados e inseguros) fue positiva buena.

El apego ansioso durante la cuarentena puede ser causado como respuesta a la exposición constante antes los cuidadores y el miedo de revertir a la normalidad previa ${ }^{(3)}$; además de ser la respuesta a la ansiedad de los cuidadores ${ }^{(17,18)}$. La evaluación del

\section{REFERENCIAS}

1. WHO. Coronavirus disease (COVID-19) outbreak [Internet]. [citado el 15 Abr. 2020]. Disponible en: https://www.who.int/emergencies/diseases/novelcoronavirus-2019

2. Fatke B, Hölzle P, Frank A, Förstl H. Psychische Probleme in der Pandemie-Beobachtungen während der COVID-19-Krise. DMW-Deutsche Medizinische Wochenschrift. 2020;145(10):675-681. doi: https//:doi.org/ 10.1055/a-1147-2889

3. Ding F, Zhang D, Cheng G. The Effect of Secure Attachment State and Infant Facial Expressions on Childless Adults' Parental Motivation. Front. Psychol. 2016;7:1237. https://doi.org/10.3389/fpsyg.2016.01237

4. Kim M, Kang S-K, Yee B, Shim S-Y, Chung M. Paternal involvement and early infant neurodevelopment: the mediation role of maternal parenting stress. BMC Pediatrics. 2016;16(1). doi: https//:doi.org/10.1186/s12887016-0747-y

5. Park S, Bellinger DC, Adamo M, Bennett B, Choi NK, Baltazar PI, et al. Mechanistic Pathways From Early Gestation Through Infancy and Neurodevelopment. Pediatrics. 2016;138(6):e20161843. doi: https//:doi.org/10.1 542/peds.2016-1843 apego ansioso cuando se revierta la cuarentena es un tópico para investigar.

Como limitante del estudio debemos destacar el escaso número de pacientes y las limitaciones del cuestionario que utilizamos para medir el nivel de ansiedad de los infantes, los acápites de ansiedad del Cuestionario de Comportamiento Infantil de 1.5 a 5 años (CBCL 1.5-5), donde estos entrelazan ansiedad y depresión.

\section{CONCLUSIONES}

Los padres/cuidadores tuvieron un alto nivel de ansiedad en los cuidadores, sin embargo, hubo solo una moderada correlación de los infantes. Sugerimos una investigación adicional en este campo que incorpore variables más diversas en una muestra mayor para mejorar nuestra comprensión del efecto Spillover en los cuidadores de la población pediátrica.

6. Johnson SB, Riis JL, Noble KG. State of the Art Review: Poverty and the Developing Brain. Pediatrics. 2016 ; 137 ( 4) : e 20153075 -e 20153075 . doi : https//:doi.org/10.1542/peds.2015-3075

7. Cariaga-Martínez A, Gutiérrez K, Alelú-Paz R. The Vast Complexity of the Epigenetic Landscape during Neurodevelopment: An Open Frame to Understanding Brain Function. Int. J. Mol. Sci. 2018;19(5):1333.

8. Yentur Doni N, Yildiz Zeyrek F, Simsek Z, Gurses G, Sahin I. Risk Factors and Relationship Between Intestinal Parasites and the Growth Retardation and Psychomotor Development Delays of Children in Şanlıurfa, Turkey. Turkiye Parazitol Derg. 2016;39(4):270-6. doi: https//:doi.org/10.5152/tpd.2015.3620

9. Kvestad I, Taneja S, Hysing M, Kumar T, Bhandari N, Strand TA. Diarrhea, Stimulation and Growth Predict Neurodevelopment in Young North Indian Children. van Wouwe J, editor. Plos One. 2015;10(3):e0121743. https//:doi.org/10.1371/journal.pone.0121743 
10. Logan AC. Dysbiotic drift: mental health, environmental grey space, and microbiota. J Physiol Anthropol. 2015;34(1). doi: https://doi.org/10.1186/s4 0101-015-0061-7

11. Berry D, Blair C, Willoughby M, Garrett-Peters P, Vernon-Feagans L, Mills-Koonce WR. Household chaos and children's cognitive and socio-emotional development in early childhood: Does childcare play a buffering role? Early Child Res. Q. 2016;34:115-27.

12. Takano T, Funahashi Y, Kaibuchi K. Neuronal Polarity: Positive and Negative Feedback Signals [Internet]. Front Cell Dev Bio. 2019 [citado el 15 Abr. 2020];7. Available from: https://www.frontiersin.org/article/10.3389/fcell.2019.0006 9/full

13. Frankenhuis WE, Nettle D, McNamara JM. Echoes of Early Life: Recent Insights From Mathematical Modeling. Child Dev. 2018;89(5):1504-1518. doi: https:/doi.org/ 10.1111/cdev.13108

14. Gao MM, Du H, Davies PT, Cummings EM. Marital Conflict Behaviors and Parenting: Dyadic Links Over Time: Marital Conflict and Parenting. Fam Relat. 2019;68(1):135-49.
15. Oliver BR, Trzaskowski M, Plomin R. Genetics of parenting: The power of the dark side. Dev Psychol. 2014;50(4):1233-40.

16. Eysenbach G. Improving the Quality of Web Surveys: The Checklist for Reporting Results of Internet E-Surveys (CHERRIES). J Med Internet Res. 2004;6(3):e34. doi: https://doi.org/10.2196/jmir.6.3.e34

17. Sherrill RB, Lochman JE, DeCoster J, Stromeyer SL. Spillover between interparental conflict and parent-child conflict within and across days. J Fam Psychol. 2017;31(7):900-9. doi: https://doi.org/10.1037/fam0000332

18. Nelson JA, O'Brien M, Blankson AN, Calkins SD, Keane SP. Family stress and parental responses to children's negative emotions: Tests of the spillover, crossover, and compensatory hypotheses. J Fam Psychol. 2009;23(5):671-9. doi: https://doi.org/10.1037/a0015977 